\title{
Upper eyelid blepharoplasty, tarsal margin rotation, and posterior lamellar super-advancement for correction of severe upper eyelid cicatricial entropion and dermatochalasis
}

Karen KW Chan ${ }^{1 *}$, MRCSEd; Chi-Lai Li ${ }^{1 *}$, FCOphthHK, FHKAM(Ophth); Regine YC Chan ${ }^{1}$, FCOphthHK, FHKAM(Ophth); Alvin L Youngl , MMedSc(Hons), FRCS(Irel); Wilson WK Yip ${ }^{l}$, FCOphthHK, FHKAM(Ophth); Kelvin KL Chong ${ }^{1,2}$, MBChB(Hons), FCOphthHK, FHKAM(Ophth) ${ }^{\prime}$ Department of Ophthalmology and Visual Sciences, Prince of Wales Hospital ${ }^{2}$ Department of Ophthalmology and Visual Sciences, The Chinese University of Hong Kong

* These authors contributed equally.

Correspondence and reprint requests:

Dr Kelvin KL Chong, Department of Ophthalmology and Visual Sciences, Prince of Wales Hospital, 30-32 Ngan Shing Street, Shatin, Hong Kong.

Email: chongkamlung@cuhk.edu.hk

\section{Abstract}

Objective: To report the long-term efficacy and safety of simultaneous correction of upper eyelid cicatricial entropion and dermatochalasis.

Method: A retrospective non-comparative chart review of consecutive patients with severe upper eyelid cicatricial entropion and dermatochalasis who underwent surgical correction from July 2012 to July 2014 at a tertiary referral center by a single surgeon.

Results: Twelve eyes of 10 patients aged $80 \pm 7$ (range, 65-92) years old received upper eyelid blepharoplasty, tarsal margin rotation, and graded posterior lamellar super-advancement. All eyes resumed normal upper eyelid margin position with no recurrence over a mean follow-up of $48.3 \pm 18$ (range, 9-70) months. Complications including suture granuloma $(n=1)$, lagophthalmos $(n=1)$, mild lid notching $(n=2)$ and residual peripheral asymptomatic trichiasis or distichiasis $(n=3)$ were managed conservatively successfully. No patients developed exposure keratopathy after surgery, and the number of lubricants required was statistically significantly reduced $(\mathrm{p}=0.005)$.

Conclusion: Our pilot study showed that combining upper lid blepharoplasty, tarsal margin rotation and graded posterior lamellar super-advancement is safe and effective in achieving long-term correction of severe upper eyelid cicatricial entropion and dermatochalasis.

Key words: Blepharoplasty; Entropion 


\section{Introduction}

Upper eyelid cicatricial entropion is a surgical challenge. It is characterized by posterior lamellar scarring, in-turning or posterior migration of mucocutaneous junction, and aberrant and misdirected lash growth causing corneal irritation and damage. Trachomatous conjunctival scarring leading to cicatricial entropion and trichiasis is the leading infectious cause of blindness worldwide. ${ }^{1}$ Other common secondary causes of cicatricial entropion include progressive scarring of upper lid tarsus and greyline owing to chronic blepharoconjunctivitis related to herpes and vernal keratoconjunctivitis, ocular cicatricial pemphigoid, StevensJohnson syndrome, longstanding use of topical glaucoma eyedrops, and previous eyelid surgeries. ${ }^{2}$

Management of cicatricial entropion is graded according to its severity (Table). ${ }^{3}$ Minimal or moderate entropion can be managed with lubricants, regular epilation, electrolysis with intrafollicular mitomycin C, cautious use of cryotherapy, margin rotation techniques, and/or everting sutures. Severe or recurrent cicatricial entropion can be surgically managed with tarsectomy, lamella repositioning, tarsal margin rotation, tarsal advancement, or posterior lamellar grafting. The reported rates of recurrence or postoperative lash misdirection (including distichiasis and/or trichiasis) ranged from $7 \%$ to $62 \% .{ }^{4}$ For posterior lamella disorder, tarsus- and lid marginbased procedures are preferred to electro-epilation. Of note, use of cryotherapy alone typically worsens the cicatricial process and is generally not recommended.

Tarsal advancement and rotation as a modification of the Trabut procedure is one of the most widely performed operations for severe upper eyelid cicatricial entropion., ${ }^{3,5}$ This method involves advancement of the posterior lamella to a position even with the inferior edge of the rotated tarsal margin. ${ }^{3}$ Later, tarsal margin rotation with modified posterior lamellar super-advancement is described, in which interlamellar dissection is carried out to the superior fornix,

\begin{tabular}{|ll|}
\hline Table. Grading of cicatricial entropion \\
\hline $\begin{array}{l}\text { Degree of } \\
\text { entropion }\end{array}$ & Clinical signs \\
\hline Minimal & $\begin{array}{l}\text { Apparent migration of meibomian glands } \\
\text { Conjunctivalization of lid margin } \\
\text { Lash-globe contact on up-gaze }\end{array}$ \\
\hline Moderate & $\begin{array}{l}\text { Apparent migration of meibomian glands } \\
\text { Conjunctivalization of lid margin }\end{array}$ \\
& $\begin{array}{l}\text { Lash-globe contact in primary position } \\
\text { Thickening of tarsal plate } \\
\text { Lid retraction }\end{array}$ \\
\hline Severe & $\begin{array}{l}\text { Gross lid distortion } \\
\text { Inwards rotation of greyline } \\
\text { Metaplastic lashes } \\
\text { Decrease or absence of upper lid margin between lashes } \\
\text { and cornea on upgaze } \\
\text { Tarsoconjunctival scarring } \\
\text { Lid retraction causing incomplete closure }\end{array}$ \\
\hline
\end{tabular}

orbital septum is opened, and levator aponeurosis is recessed, after which the posterior lamella is advanced and fixated beyond the inferior edge of the rotated margin for 2 to $3 \mathrm{~mm} .^{6}$ This modification is useful for severe or recurrent cases that have failed previous surgical correction. The tarsal margin is rotated outwards for $180^{\circ}$ and a new upper eyelid margin, the 'neo-greyline', is formed in good position. However, in our experience, eyelash ptosis secondary to relative redundancy of anterior lamella may occur, especially in older patients with mild degree of dermatochalasis (Figure 1). This leads to recurrent lash-globe contact and keratopathy, requiring further surgeries including upper eyelid blepharoplasty with or without buried everting sutures and/or electrolysis.

Techniques to prevent downsliding of anterior lamella include anterior lamellar fixation sutures, ${ }^{3,7}$ skin grafting, ${ }^{8}$ buccal mucosal membrane grafting, ${ }^{9}$ and combination of blepharoplasty to anterior lamellar repositioning with complete lid split or bilamellar tarsal rotation. ${ }^{10,11}$ We propose a one-stage surgery combining an established cicatricial entropion correction procedure using tarsal margin rotation and graded posterior lamellar super-advancement, with the addition of skin-muscle removing blepharoplasty, for older patients with severe upper eyelid cicatricial entropion and established or early dermatochalasis. We aim to report on the long-term efficacy and safety of this one-stage surgical technique that combines three established surgical procedures in a graded fashion.

\section{Methods}

The study adhered to the tenets of the Declaration of Helsinki.

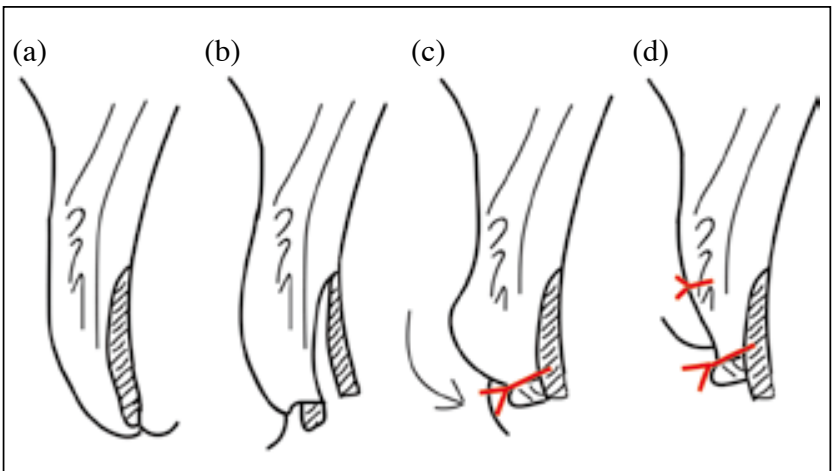

Figure 1. Schematic diagram showing (a) preoperative appearance of cicatricial entropion with internal rotation of eyelash (tarsus represented by shaded area). (b) Intraoperative posterior tarsotomy and interlamellar dissection distally into lash roots and proximally until superior border of tarsus with levator aponeurosis disinsertion, with $180^{\circ}$ rotation of the inferior tarsal segment. (c) Traditional tarsal advancement and rotation alone may lead to postoperative anterior lamellar redundancy and secondary lash ptosis. (d) Blepharoplasty combined with tarsal margin rotation, anterior lamellar recession and posterior lamellar super-advancement creates a new eyelid margin and provides a platform to prevent anterior lamellar internal rotation and metaplastic lashes from contacting with the globe 
The study was approved by the Joint Chinese University of Hong Kong-New Territories East Cluster Clinical Research Ethics Committee (CREC 2017.151). Medical records of consecutive patients with upper eyelid cicatricial entropion and dermatochalasis who underwent triple surgical procedures at a tertiary referral center from July 2012 to July 2014 were retrospectively reviewed. Indications for surgery included severe upper eyelid cicatricial entropion defined by lash-globe contact in primary gaze, decrease or absence of upper lid margin between the lashes and cornea on upgaze, ${ }^{12}$ associated tarsoconjunctival scarring, and inward rotation of the mucocutaneous junction of greyline, with or without metaplastic lashes. ${ }^{3}$

Digital photographs of the eyelids in primary gaze and side view were taken before and after surgery for each patient. Visual acuity, slit-lamp examinations, surgery-related complications, and use of lubricants before and after surgery were compared to assess outcomes.

Primary outcome was surgical success, defined as correction of upper eyelid entropion with no lash-globe contact in primary gaze, without the need for postoperative epilation or further lash procedures. Surgery-related complications, if any, were recorded.

Statistical analyses were performed using SPSS (version 24.0; IBM, Armonk [NY], USA). Continuous variables were reported as mean \pm standard deviation, and unpaired t-test was used to test for their group differences. Categorical data were expressed in frequencies and percentages. $\mathrm{P}$ values of $<0.05$ were considered statistically significant.

\section{Surgical Technique}

An upper eyelid blepharoplasty with removal of skin and orbicularis muscle was performed first. Preoperative skin markings were made with the patient sitting upright looking in primary gaze. The lower skin marking was set $4-5 \mathrm{~mm}$ above the cilia margin for bilateral operations or at the level of the upper lid crease of the fellow eye for unilateral cases. The upper marking should be at least $15 \mathrm{~mm}$ inferior to the lower border of the eyebrow. A skin pinch test was used to adjust preoperative markings and to minimize lagophthalmos. Surgery started with standard aseptic prepping and draping after infiltration of $3 \mathrm{ml}$ of local anaesthetic solution, $2 \%$ xylocaine with 1:200,000 adrenaline (Aspen Pharma, Ireland), into the greyline, subcutaneous and submuscular planes. Skin was then incised using a No.15 Park blade. Skin and a thin strip of orbicularis was removed using monopolar or handheld hot-tip cautery keeping the orbital septum intact. Upper eyelid was then everted over a Desmarres eyelid retractor (Figure 2b). An incision was made through the subtarsal sulcus or where the Arlt's line was present with a No.11 blade (Figure 2c). A full-thickness tarsotomy was completed across the entire eyelid horizontal length $1 \mathrm{~mm}$ short of the upper eyelid punctum to the lateral canthal angle (Figure 2d). The lower tarsal segment was dissected off the pretarsal orbicularis until the lash roots can be seen (Figure 2e). Importantly, relaxing vertical cuts were made at both ends so that the lower tarsal segment was rotated $180^{\circ}$ along with the original greyline (Figure 2f). At this point, all lashes should be pointing up and away from the ocular surface. Dissection between the anterior and posterior lamellae proceeded upwards from the tarsotomy incision to the superior border of the upper tarsus (Figure 1b). The levator aponeurosis was then recessed in a graded manner, titrated to the amount of lagophthalmos or upper lid retraction during surgery. The Muller's muscle was not disturbed. The anterior lamella including the pretarsal skin and rotated inferior tarsal segment was recessed upwards, while the posterior lamella was recessed downwards and super-advanced $2 \mathrm{~mm}$ beyond the edge of the rotated tarsal margin to form the "neo-greyline" (Figure 2g-2i). On-table adjustments were made by asking patients to open their eyes and look straight ahead towards the ceiling. Any difficulty in posterior lamellar super-advancement and contour deformity of each lamella or segment was then released in a graded fashion. The repositioned anterior and super-advanced posterior lamellae were then fixed with 3 pairs of double-arm 4/0 silk sutures placed horizontally (Figure $\mathbf{2 j}$ ), entering the pretarsal skin of the rotated lash line full-thickness, then across the superior tarsus in a lamellar fashion, and finally exiting and tied over the recessed anterior lamella (Figure $\mathbf{2 k}$ and $\mathbf{1 d}$ ). Each everting suture should be pre-placed and left long before tying, with the tension adjusted to avoid buckling of the tarsus. The posterior lamella was super-advanced $2 \mathrm{~mm}$ showing bare tarsus, and this raw mucosal surface was left to granulate in 4-6 weeks. Finally, the blepharoplasty wound was closed with interrupted $6 / 0$ vicryl or dermalon sutures (Figure 2l).

Eyes were dressed with ointment Maxitrol (Novartis, United Kingdom) and double-padded for 1 day. Follow-up examination was scheduled at 1 week, 1 month, 3 months, and 6 months, with annual follow-up thereafter. Nonabsorbable blepharoplasty sutures were removed after 1 week. Silk sutures fixating the anterior and posterior lamellae were removed at 4 weeks.

\section{Results}

Medical records of 12 eyes (6 left and 6 right) from five male and five female consecutive patients aged 65 to 92 (mean $\pm \mathrm{SD}, 80.1 \pm 7$ ) years who underwent the combined triple techniques for severe upper eyelid cicatricial entropion and dermatochalasis by a single oculoplastic surgeon were reviewed.

All 12 upper eyelids had trichiasis or metaplastic lashes involving over half of the upper eyelid, and concomitant dermatochalasis. Four (33.3\%) eyes had a history of cryosurgical epilation, and two (16.7\%) eyes had a history of folliculectomy for trichiasis. One $(8.3 \%)$ patient had undergone the Weis procedure elsewhere.$^{13}$ Definite Arlt's line was noted during surgery in $4(33.3 \%)$ eyes. No patient had documented history of herpetic infection, ocular cicatricial pemphigoid, Stevens-Johnson syndrome, or was on topical anti-glaucomatous. There was no preoperative lid retraction 


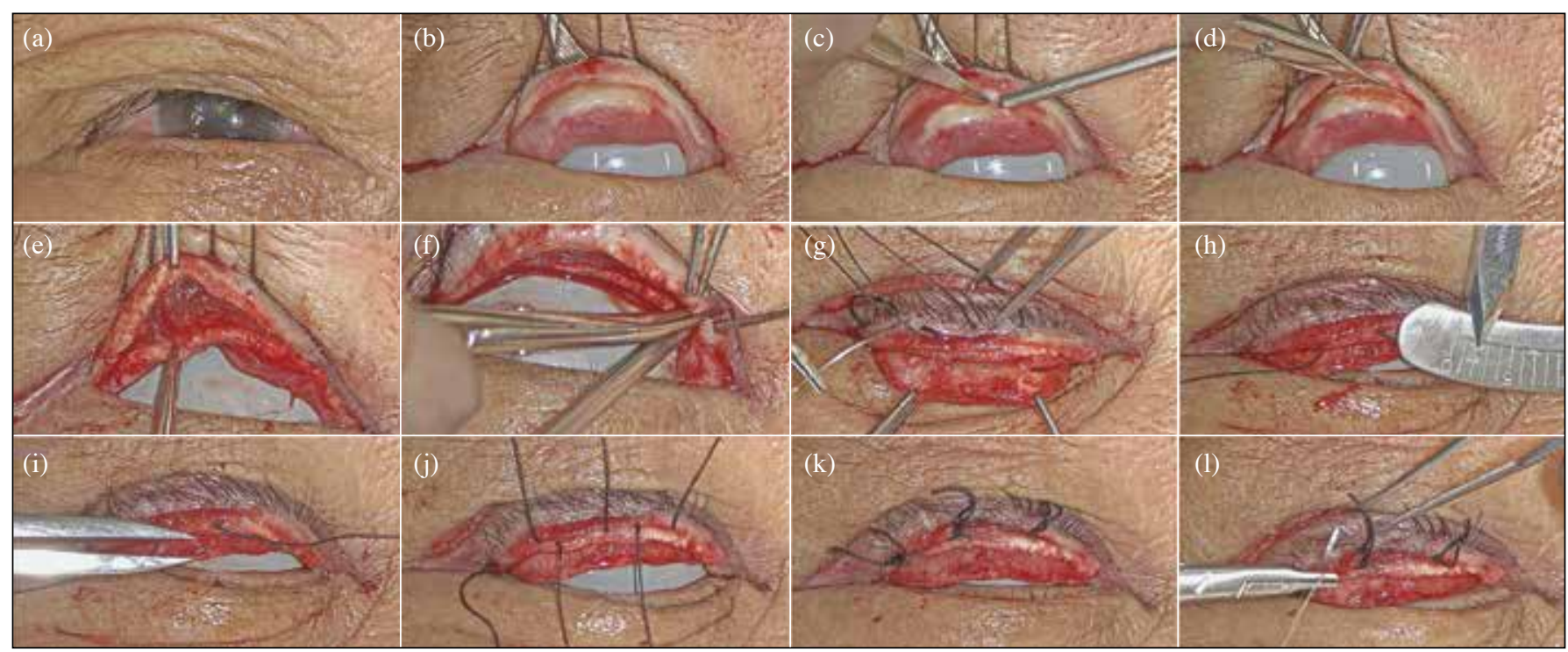

Figure 2. Intraoperative photographs demonstrating the triple surgical technique: (a) Preoperative appearance with severe upper lid entropion and dermatochalasis. (b) After an upper lid blepharoplasty, the upper eyelid was everted over a Desmarres eyelid retractor after placement of Frost sutures and corneal protector. (c) An incision was made at the subtarsal sulcus or Arlt's line (if present) with a No.11 blade. (d) A full thickness tarsotomy was completed across the entire eyelid horizontal length with sharp Westcott scissors. (e) The lower tarsal segment was dissected off the pretarsal orbicularis until the lash roots can be seen. (f) Relaxing vertical cuts were made at both ends. (g) Dissection was further proceeded upwards from the tarsotomy incision to the superior border of the upper tarsus to disinsert the levator aponeurosis. The anterior lamella including the pretarsal skin and 180-degrees rotated inferior tarsal segment was then recessed upwards, while the posterior lamella was pulled downwards. (h, i) The posterior lamella was super-advanced $2 \mathrm{~mm}$ (measured with a calliper) beyond the edge of the rotated tarsal margin to form the "neo-greyline". $(j, k)$ The repositioned anterior and posterior lamellae were fixed with 3 pairs of horizontally placed $4 / 0$ silk sutures. (l) Finally, the blepharoplasty wound was closed with interrupted $6 / 0$ vicryl sutures

or lagophthalmos. Five (41.7\%) eyes had pre-existing dense corneal scars.

The mean surgery duration was $50 \pm 14$ (range, 35-84) minutes per eye. After a mean follow-up duration of $48.3 \pm 18$ (range, 9-70) months, all patients maintained normal upper lid position and improvement of symptoms. Three (25\%) patients had residual non-central, asymptomatic trichiasis or distichiasis that was managed on self-epilation, and did not require further surgery. One $(8.3 \%)$ patient developed suture granuloma that was removed at 2 months after surgery. One $(8.3 \%)$ patient had lagophthalmos that resolved conservatively after 4 months. Two (16.7\%) eyes had mild lid notching that did not require revisional surgery. No eyes developed lid necrosis, worsening of keratopathy, or exposure keratopathy. None of the patients with unilateral treatment received blepharoplasty in the fellow eye, as these patients mostly sought for functional correction rather than eyelid symmetry cosmetically. Following surgery, visual acuity was similar $(\mathrm{p}=0.58)$, but the number of lubricants required reduced significantly $(\mathrm{p}=0.005)$.

\section{Discussion}

Severe upper eyelid cicatricial entropion is difficult to treat. Recurrence is common, and postoperative adjunctive lash destruction procedures are frequently needed. Bilamellar tarsal rotation or posterior lamellar tarsal rotation procedures with various modifications are the most commonly used methods, ${ }^{14,15}$ whereas tarsal advancement or posterior lamellar lengthening procedures are used to treat severe cicatricial entropion with posterior lamellar lid shortening. ${ }^{3}$

Although technically challenging, we found that tarsal margin rotation combined with posterior lamellar advancement is effective in severe upper eyelid cicatricial entropion with or without pre-existing upper eyelid retraction. Posterior tarsotomy and marginal rotation divert metaplastic cilia at the entropic upper eyelid margin $180^{\circ}$ away from the corneal surface. ${ }^{3}$ Any subsequent metaplastic lash or distichiasis can then be easily treated by lash ablation. In addition, superadvancement of the posterior lamellar $2 \mathrm{~mm}$ beyond the anterior lamella provides an additional mucosal platform to mechanically shield the upper lid margin and lashes from the cornea. ${ }^{6}$ This method can also avoid posterior lamella grafting and associated problems of donor site morbidities, harvesting, and unpredictable graft survival. ${ }^{6}$ In eyes with significant tarsal shortening, additional posterior lamellar grafting using buccal mucosa or allogenic material is needed to maintain upper lid stability.

Long-term failure of tarsal margin rotation combined with posterior lamellar advancement has been reported., ${ }^{3,12}$ The underlying mechanism of late recurrence is not well 


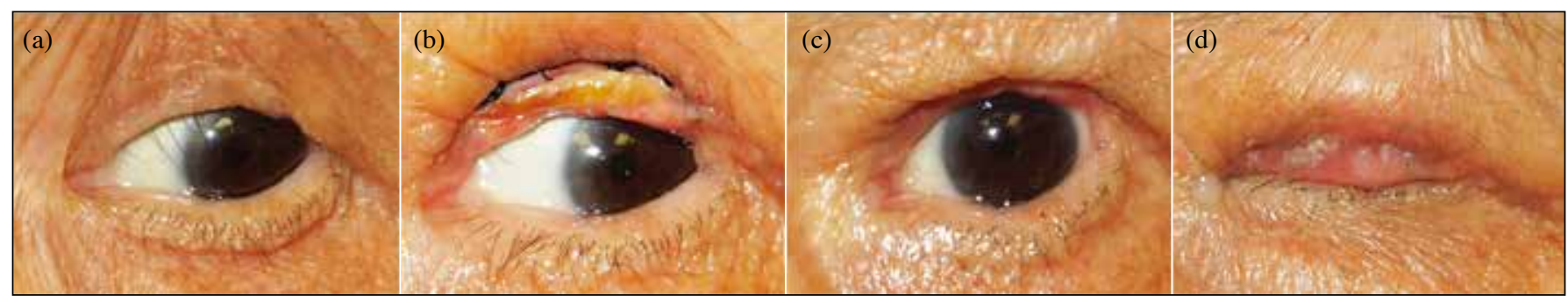

Figure 3. Pre- and postoperative appearance of a patient who received triple surgical technique. (a) Preoperative assessment with severe upper eyelid entropion and dermatochalasis. (b) One-week postoperative appearance. Her "neo-greyline" was left to granulate, and silk sutures holding the anterior and posterior lamellae were left in-situ for 4 weeks. (c) Two-months postoperative photograph showing formation of new eyelid margin with no recurrence. (d) Two-months postoperative appearance on eyelid closure with absence of lagophthalmos

defined and likely related to ongoing cicatricial process. Our experience with tarsal margin rotation and posterior lamellar super-advancement was favourable although ocular irritation may develop over time due to lash ptosis or metaplasia, which required further eyelash everting sutures or simple electrolysis. We believe this is related to relative anterior lamella redundancy after lengthening by the rotated distal tarsal segment. Skin redundancy after tarsal margin rotation together with inadequate posterior lamellar advancement lead to lash ptosis and corneal irritation (Figure 1c). In our cohort, additional upper lid blepharoplasty allowed anterior lamella to be shortened and repositioned upwards more effectively. Postoperative anterior lamellar redundancy and secondary eyelash ptosis was minimised (Figure 1d). Addition of preaponeurotic fat debulking may be considered in Asian lids, which have a lower insertion of the levator aponeurosis to the orbital septum with consequent lower descent of preaponeurotic fat. ${ }^{16}$ Further studies are warranted to evaluate this surgical adjunct.

Marginal tarsal rotation induces upper eyelid retraction due to posterior lamellar shortening. Treatment of upper eyelid cicatricial entropion associated with lid retraction without posterior lamellar grafting depend on adequate posterior lamellar super-advancement which in turn is related to interlamellar dissection and levator aponeurosis disinsertion or recession. Our follow-up results showed that patients usually exhibited very mild postoperative lid retraction after graded recession or disinsertion of levator aponeurosis from the tarsal plate for posterior lamella advancement. This is compatible to findings in a study of terminal tarsal rotation with posterior lamellar advancement that leads to a significant increase in mean palpebral aperture from $10.1 \mathrm{~mm}$ to $11.5 \mathrm{~mm},{ }^{17}$ suggesting surgically induced upper lid retraction. In our series, lagophthalmos developed in one (8.3\%) eye and resolved in 4 months. No patient developed exposure keratopathy despite mild postoperative lid retraction (Figures 3c). From our results, interlamellar dissection upwards towards the superior fornix may not be necessary. ${ }^{6}$ Rather, dissection up to the superior tarsal border with graded disinsertion of the levator aponeurosis is adequate as verified during surgery.
The neo-greyline with exposed tarsus appears inflamed immediately after surgery and can be alarming to patients (Figure 3b). However, it heals with granulation and epidermalization around 2 months after surgery with excellent cosmetic outcomes (Figure 3c) ${ }^{6}$ Therefore, it is good practice to counsel patients on these expected surgical outcomes before surgery for better preparation and acceptance of initial cosmetic results.

\section{Conclusion}

The one-stage combined skin-muscle removing blepharoplasty, tarsal margin rotation, and posterior lamellar super-advancement in a graded fashion can achieve sustained correction in patients with severe upper eyelid cicatricial entropion and dermatochalasis, minimizing recurrence or need of postoperative adjunctive eyelash destruction procedures.

\section{Author contributions}

Concept or design: CLL, KKLC

Acquisition of data: KKWC, CLL

Analysis or interpretation of data: KKWC, CLL

Drafting of the article: KKWC, CLL, KKLC

Critical revision for important intellectual content: All authors

All authors had full access to the data, contributed to the study, approved the final version for publication, and take responsibility for its accuracy and integrity.

\section{Conflicts of interest}

As editors of the Journal, KKL Chong and A Young were not involved in the peer review process for this article. Other authors have disclosed no conflicts of interest.

\section{Funding/support}

This research received no specific grant from any funding agency in the public, commercial, or not-for-profit sectors. 


\section{Ethics approval}

This study was approved by the Joint Chinese University of Hong Kong-New Territories East Cluster Clinical Research
Ethics Committee (Ref CREC 2017.151). The need for patient consent was waived. All patients provided informed consent for all treatments and procedures. Patients photographed in figures 2 and 3 provided written consent for publication.

\section{References}

1. World Health Organization. Trachoma 2020 [updated Jan 2020. Available from: https://www.who.int/news-room/factsheets/detail/trachoma.

2. Chi M, Kim HJ, Vagefi R, Kersten RC. Modified tarsotomy for the treatment of severe cicatricial entropion. Eye (Lond) 2016;30:992-7. Crossref

3. Kemp EG, Collin JR. Surgical management of upper lid entropion. Br J Ophthalmol 1986;70:575-9. Crossref

4. Rajak SN, Collin JR, Burton MJ. Trachomatous trichiasis and its management in endemic countries. Surv Ophthalmol 2012;57:105-35. Crossref

5. Trabut G. Entropion-trichiasis en Afrique du Nord. Arch Ophthalmol (Paris) 1949;9:701-7.

6. Seiff SR, Carter SR, Tovilla y Canales JL, Choo PH. Tarsal margin rotation with posterior lamella superadvancement for the management of cicatricial entropion of the upper eyelid. Am J Ophthalmol 1999;127:67-71. Crossref

7. Aghai GH, Gordiz A, Falavarjani KG, Kashkouli MB. Anterior lamellar recession, blepharoplasty, and supratarsal fixation for cicatricial upper eyelid entropion without lagophthalmos. Eye (Lond) 2016;30:627-31. Crossref

8. Steinkogler FJ. Treatment of upper eyelid entropion. Lid split surgery and fibrin sealing of free skin transplants. Ophthalmic Plast Reconstr Surg. 1986;2:183-7. Crossref

9. Koreen IV, Taich A, Elner VM. Anterior lamellar recession with buccal mucous membrane grafting for cicatricial entropion. Ophthalmic Plast Reconstr Surg 2009;25:180-4. Crossref

10. Bi YL, Zhou $Q, X u W$, Rong A. Anterior lamellar repositioning with complete lid split: a modified method for treating upper eyelids trichiasis in Asian patients. J Plast Reconstr Aesthet Surg 2009;62:1395-402. Crossref

11. Sadiq MN, Pai A. Management of trachomatous cicatricial entropion of the upper eye lid: our modified technique. J Ayub Med Coll Abbottabad 2005;17:1-4.

12. Reacher MH, Muñoz B, Alghassany A, Daar AS, Elbualy M, Taylor HR. A controlled trial of surgery for trachomatous trichiasis of the upper lid. Arch Ophthalmol 1992;110:66774. Crossref

13. Wies FA. Spastic entropion. Trans Am Acad Ophthalmol Otolaryngol 1955;59:503-6.

14. Reacher M, Foster A, Huber J, Blindness WHOPftPo. Trichiasis surgery for trachoma: the bilamellar tarsal rotation procedure. Geneva: World Health Organization; 1993.

15. Kettesy A. On genesis and operation of the cicatricial (trachomatous) entropion of the upper lid. Br J Ophthalmol 1948;32:419-23. Crossref

16. Tyers AG, Collin JRO. Color Atlas of Ophthalmic Plastic Surgery. 4th ed. Elsevier; 2017.

17. Dhaliwal U, Monga PK, Gupta VP. Comparison of three surgical procedures of differing complexity in the correction of trachomatous upper lid entropion: a prospective study. Orbit 2004;23:227-36. Crossref 